\title{
EL MODELO TRANSTEÓRICO APLICADO AL CAMBIO DE CONDUCTAS RELACIONADAS CON LA REDUCCIÓN DEL PESO CORPORAL
}

\author{
Enrique Berra Ruiz y Sandra Ivonne Muñoz Maldonado \\ UABC, UNAM \\ México
}

\begin{abstract}
RESUMEN
El ritmo de vida actual ha generado diversos cambios en los estilos de vida saludables; por ello la Psicología de la Salud ha enfocado sus esfuerzos en el manejo de conductas de riesgo como medida preventiva o de intervención. El modelo transteórico es una de las propuestas que ha incorporado diversos elementos basados en la intención del cambio y la motivación, para facilitar el cambio de conducta teniendo en cuenta un proceso en etapas. Inicialmente este modelo fue usado para atender conductas adictivas, pero se ha considerado útil para modificar muchos otros comportamientos que representen un riesgo para la salud. Este trabajo describe las variables del modelo y se ejemplifica su aplicación en la evaluación e intervención de sobrepeso y obesidad.
\end{abstract}

\section{Palabras Clave:}

modelo transteórico, cambio, obesidad, sobrepeso, conductas de riesgo.

\section{TRANSHEORETICAL MODEL APPLIED TO THE CHANGE OF BEHAVIORS RELATED TO THE REDUCTION OF BODY WEIGHT}

\begin{abstract}
The current rhythm of life has generated various changes in healthy lifestyles, which is why health psychology has focused its efforts on changing risk behaviors as a preventive measure or intervention. The Transtheorical Model is one of the proposals that has incorporated various elements based on the intention of change and motivation, to facilitate behavior change contemplating a process in stages. This model was initially used to address addictive behaviors but has been found useful for modifying many other behaviors that pose a health risk. This paper describes the variables of the model and exemplifies its application in the evaluation and intervention of overweight and obesity.
\end{abstract}

Keywords:

transtheorical model, change, obesity, overweight, risk behaviors

Bitácora del Artículo:

| Recibido: 24 de Enero de 2018 | Aceptado: Noviembre de 2018 | Publicado en línea: Julio-Diciembre de 2018 | 


\title{
Autoría y Derechos de Propiedad Intelectual
}

\section{EL MODELO TRANSTEÓRICO APLICADO AL CAMBIO DE CONDUCTAS RELACIONADAS CON LA REDUCCIÓN DEL PESO CORPORAL}

\author{
Enrique Berra Ruiz y Sandra Ivonne Muñoz Maldonado \\ UABC, UNAM \\ México
}

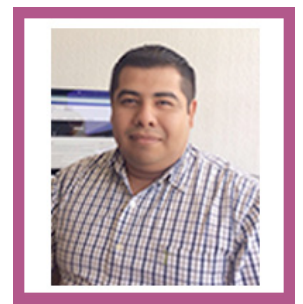

Enrique Berra Ruiz

ECISALUD-UABC, FES-Iztacala, UNAM

Correo: enrique.berra@uabc.edu.mx

Doctor en Psicología de la Salud por la UNAM. Es profesor de T.C. en la Escuela de Ciencias de la Salud de la Universidad Autónoma de Baja California y profesor de asignatura en la licenciatura en Psicología del SUAYED en la FES Iztacala. Sus trabajos se enfocan en temas relacionados con estrés y salud, entre otros.

Ver más...

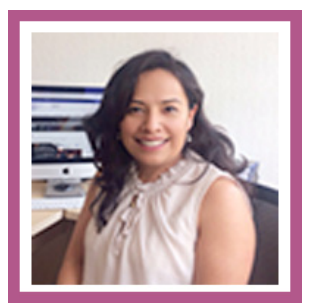

Sandra Ivonne Muñoz Maldonado FES-Iztacala, UNAM

Correo: sandra.munoz@ired.unam.mx

Doctora en Psicología y Salud por la UNAM. Profesora de la licenciatura en Psicología a distancia en la FESIztacala.(SUAyED-UNAM), en las áreas de Psicología de la Salud y Psicología Clínica. Sus líneas de investigación se enfocan en problemas de obesidad adolescente, su relación con emociones, por mencionar algunas. Ver más...

\section{CONTRIBUCIÓN DE LOS AUTORES}

Enrique Berra Ruiz coordinó la integración del documento, construyó el esquema y participó en la redacción del artículo "El modelo transteórico aplicado al cambio de conductas relacionadas con la reducción del peso corporal" | Sandra Ivonne Muñoz Maldonado construyó el esquema y participó en la redacción del artículo "El modelo transteórico aplicado al cambio de conductas relacionadas con la reducción del peso corporal.

\section{AGRADECIMIENTOS}

[Agradecimiento por parte de los autores a intituciones, personas, etc. participantes en el estudio.]

\section{Datos de FiLIACIÓN DE los Autores}

Facultad de Estudios Superiores Iztacala, UNAM.

\section{(c) (9)}

Copyright: ( 2018 Berra-Ruiz, E. \& Muñoz-Maldonado, S.I.

Este es un artículo de acceso abierto distribuido bajo los términos de la licencia Creative Commons Reconocimiento-NoComercial 4.0 Internacional, por lo que su contenido gráfico y escrito se puede compartir, copiar y redistribuir total o parcialmente sin necesidad de permiso expreso de sus autores con la única condición de que no se puede usar con fines directamente comerciales y los términos legales de cualquier trabajo derivado deben ser los mismos que se expresan en la presente declaración. La única condición es que se cite la fuente con referencia a la Revista Digital Internacional de Psicología y Ciencia Social y a sus autoras. 


\section{TABLA DE CONTENIDO}

INTRODUCCIÓN

Antecedentes conceptuales del modelo teórico, 24

El modelo transteórico: procesos, estadios y niveles de cambio, 25 


\section{INTRODUCCIÓN}

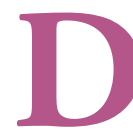
urante casi cuatro décadas el modelo transteórico (MT) ha planteado que el cambio comportamental es un proceso en el que las personas tienen diversos niveles de motivación y de intención para modificar su conducta; conformado por una serie de postulados respecto a la naturaleza del ajuste de comportamiento que hace el individuo y de las características de las intervenciones que pueden facilitar dicha modificación. De acuerdo con Cabrera (2000), el análisis comparativo de 29 teorías relevantes, y disponibles a finales de los años setenta hecho por Prochaska, permitió iniciar los trabajos de este modelo con consumidores de drogas ilegales y tabaco que después se trasladaron al trabajo con las conductas asociadas a comportamientos promotores de salud.

Las investigaciones recientes en distintas poblaciones han aportado evidencia del uso del MT en comportamientos de riesgo, como fumar (Gökbayrak, Paiva, Blissmer y Prochaska, 2015; Guo, Chang, Fu y Hsu, 2016),o consumo excesivo de alcohol (Crouch, DiClemente y Pitts, 2015), logrando reducir el consumo de cigarros y alcohol a corto plazo, y en los seguimientos a seis meses.

Estos logros se han trasladado a otras conductas relacionadas con enfermedades como la obesidad, que de acuerdo con la OMS (2017) se asocia con una amplia gama de padecimientos que amenazan la salud de los individuos, como los problemas cardiovasculares, diabetes y cáncer, entre otros, que a la postre implica gastos en salud significativos para los individuos y los gobiernos. Dentro de las acciones que la OMS han sugerido para disminuir el impacto de este tipo de enfermedades está el disminuir peso corporal, por medio de una mayor actividad física para incrementar el gasto energético, la adopción de una dieta equilibrada que implique un incremento en el consumo de verduras, frutas, agua simple, así como la disminución de comida rica en grasas, carbohidratos y sales.

En particular se ha identificado que aspectos como la autoeficacia, el balance decisional y la entrevista motivacional son un apoyo para la intervención, porque al incorporarlos permiten reforzar la capacidad de cambio en las etapas y procesos, facilitando comportamientos saludables como el consumo de alimentos saludables y la adopción de prácticas deportivas regulares. Esto permite obtener una gran ventaja para diseñar acciones dirigidas a los individuos teniendo en cuenta su nivel de motivación, la autoeficacia percibida, las barreras y beneficios identificados, es decir, al identificar o distinguir de modo apropiado la etapa de cambio en que se encuentra cada persona e incorporar habilidades especiales, proporcionará mayor motivación para intentar el nuevo comportamiento, y con ello incrementar la confianza a su propia eficacia; se han encontrado resultados de intervenciones que muestran tendencias significativas a los cambios esperados por etapa de cambio (Rojas-Russell, 2009; Márquez-Ibáñez, Armendáriz-Anguiano, Bacardí-Gascón y Jiménez-Cruz, 2008; Sánchez, García y Landabaso,1998; Coombes y McPherson, 1996).

Ante este panorama, el modelo transteórico (Prochaska, 2013; Prochaska et al., 1994) es un marco conceptual de cambio conductual que ha resultado efectivo para programas de control de peso, ayudando a los individuos a identificar su disposición al cambio de conductas como el aumento del consumo de alimentos saludables y la actividad física, lo cual finalmente impacta en una disminución de peso (Greene et al., 2013; Lipschitz et al., 2015; Spencer, Wharton, Moyle y Adams, 2007).

El objetivo del presente artículo es ejemplificar los elementos del MT en conductas relacionadas con la reducción de peso, como la adherencia a una dieta sana y el incremento de actividad física, que son comportamientos protectores para el desarrollo de obesidad y enfermedades no trasmisibles, como la diabetes y el cáncer.

En primer lugar el escrito presentará los antecedentes del MT; en seguida describirá los procesos de cambio, etapas de cambio y niveles de cambio, para después ejemplificar cada uno de ellos en relación con comportamientos asociados con el peso corporal, y por último se presentan algunas conclusiones respecto al uso del MT para la intervención en problemas de salud en donde es necesario mejorar la actividad física y la dieta.

\section{Antecedentes conceptuales del modelo teórico}

El modelo transteórico del cambio del comportamiento fue propuesto a principios de 1980 por James Prochaska y Carlo DiClemente como resultado de la integración de los principios y componentes de diferentes intervenciones para explicar los cambios en las personas con adicciones, considerando las siguientes premisas para establecer el modelo (Prochaska y DiClemente, como se cita en Díaz, 2001):

1) Considera un amplio rango de maneras de cambio: desde intervenciones tradicionales de rehabilitación hasta la automodificación del comportamiento sin ayuda especializada. 
2) Logra aplicar a las diversas conductas adictivas: desde el abuso de alcohol y la dependencia a cualquier sustancia (nicotina, heroína, cocaína, etcétera) hasta comportamientos adictivos no farmacológicos, como la hiperfagia o adicción al juego.

3) Integra los distintos modelos de cambio que estaban disponibles en aquel momento para las conductas problemáticas: sobre todo los que proponen una explicación global del cambio.

4) Aborda todo el proceso de cambio: desde que el individuo comienza a percibir su comportamiento como problemático hasta que el problema deja de existir.

5) Considera los avances de investigación, incorporando e integrando las variables que se comprueben significativas en la explicación de la modificación de conductas en las personas.

Respecto al MT, Díaz (2001) establece que esta propuesta no debe considerarse como una visión ecléctica, dado que no es una mezcla pragmática de procedimientos, sino que éste pretende integrar las teorías subyacentes a las psicoterapias y no sólo las técnicas, lo que considera como flexibilidad teórica.

La premisa principal de este modelo es el cambio, que es concebido como un proceso en el cual las personas pueden tener diversos niveles de motivación e intención de modificarlo, lo que permite aplicarlo a cualquier conducta y en cualquier contexto, porque la intervención se hace de manera individualizada teniendo en cuenta determinada influencia externa y un claro compromiso personal que puede ubicarse en alguna etapa que plantea el modelo, y a partir de ésta implementar los procesos de acuerdo con el nivel que deseen intervenir para modificar y solucionar el problema (figura 1).

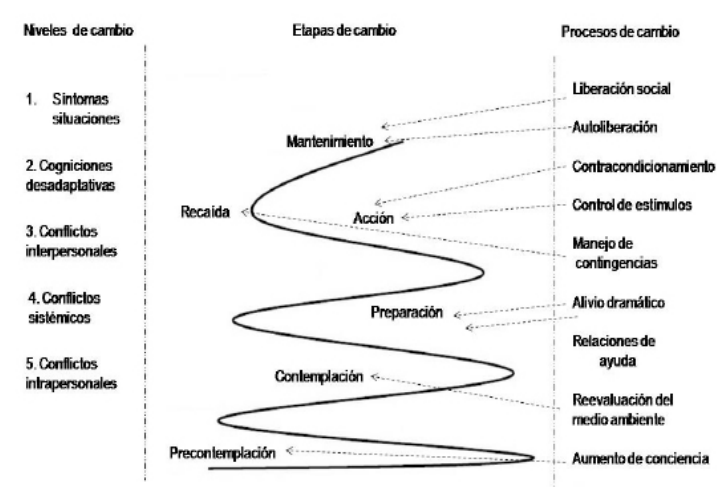

Figura 1.

Elementos del modelo transteórico del cambio de Prochaska y DiClemente (1982). Adaptada de Prochaska, DiClemente y Norcross (1992), p. 1108.

\section{El modelo transteórico: procesos, estadios y niveles de cambio}

Con base en los estudios y análisis teóricos de Prochaska a finales de 1960 respecto a los distintos enfoques teóricos e intervenciones psicológicas, Prochaska, Norcross y DiClemente (2013) identificaron la estructura del cambio intencional del comportamiento que explican mediante tres dimensiones (procesos, estadios y niveles de cambio).

En primer lugar se encuentran los estadios de cambio, que son fases que pueden ser identificadas en el individuo y permiten planear la intervención de acuerdo con el momento en que se encuentra (tabla 1).

Un aspecto relevante es que el curso en estos estadios no es lineal; además se pueden presentar las recaídas que ocurren cuando las acciones de cambio fracasan para continuar en la fase de acción o mantenimiento, causando un retroceso hacia estadios previos. Cuando se logran estabilizar los cambios y mantener los nuevos hábitos, se puede considerar un estadio de finalización, en el que se considera la extinción de la conducta problema o de las tentaciones para realizar las conductas de riesgo.

En segundo lugar, el modelo plantea los procesos de cambio definidos por Prochaska, DiClemente y Norcross (1992) como "las actividades abiertas o encubiertas y experiencias en las que los individuos se involucran cuando intentan modificar comportamientos problemáticos" (p. 1107); una definición más amplia del proceso de cambio es la que refiere Díaz (2001): "aquellas actividades abiertas o encubiertas en las que los individuos se implican para modificar sentimientos, pensamientos, conductas o relaciones interpersonales relativos a conductas o estilos de vida problemáticos" (p. 24).

De acuerdo con Prochaska et al. (1992), cada proceso puede abarcar múltiples técnicas y métodos de cambio recomendados en diferentes teorías, lo que explicaría el término "transteórico". Los procesos pueden incorporarse en las sesiones de terapia; entre las sesiones o sin las sesiones de terapia, los 10 procesos principales que han producido patrones similares se muestran en la tabla 2, aunque también se han organizado en dos factores de segundo orden: 1) procesos de cambio cognitivos (aumento de conciencia, autorreevaluación, reevaluación ambiental, alivio dramático y liberación social), y 2) procesos de cambios conductuales (autoliberación, manejo de contingencias, relaciones de ayuda, contracondicionamiento y control de estímulos).

En tercer lugar, el modelo contempla los niveles de cambio, que se refieren al contenido específico del cambio, las conductas a las que el individuo aplica los procesos de cambio con el objetivo de modificarlas (Díaz, 2001). Esta dimensión, sobre todo planteada en el mode- 
Tabla 1.

Explicación de los estadios de cambio y ejemplos

\begin{tabular}{|c|c|c|}
\hline ETAPA & DESCRIPCIÓN & EJEMPLO \\
\hline Precontemplación & $\begin{array}{l}\text { Es la etapa en que no hay intención de cambiar el } \\
\text { comportamiento en el futuro previsible. Muchos } \\
\text { individuos en esta etapa no son conscientes de } \\
\text { sus problemas. No se trata de que no puedan } \\
\text { hallar una solución, sino que no pueden ver el } \\
\text { problema. Esta es una de las razones por la que } \\
\text { no acuden a tratamiento, o si lo hacen es por estar } \\
\text { presionados por alguna circunstancia ambiental, } \\
\text { abandonando el cambio al disminuir dicha presión. }\end{array}$ & $\begin{array}{l}\text { Es la etapa en que están las personas que } \\
\text { observan un incremento en su peso, pero que } \\
\text { no pueden identificar el riesgo para su salud en } \\
\text { esta condición. En mayor medida puede estar } \\
\text { asociada con creencias erróneas, como a mayor } \\
\text { peso una mejor alimentación, y por tanto no se } \\
\text { presentan una intención de cambio. }\end{array}$ \\
\hline Contemplación & $\begin{array}{l}\text { Es la etapa en que las personas reconocen que } \\
\text { existe un problema y piensan seriamente en su } \\
\text { superación, pero aún no se han comprometido a } \\
\text { tomar medidas. Las personas pueden permanecer } \\
\text { atrapadas en esta etapa durante largos periodos; } \\
\text { están más abiertas a la información acerca de su } \\
\text { comportamiento. }\end{array}$ & $\begin{array}{l}\text { Se pueden situar a las personas que se } \\
\text { encuentran con un horario de trabajo absorbente } \\
\text { que no les permite llevar una alimentación } \\
\text { equilibrada, lo que implica generar un problema } \\
\text { de obesidad y ante esto comienzan a identificar } \\
\text { insatisfacción en su apariencia o en su condición } \\
\text { física, generando motivación para el cambio, aun } \\
\text { cuando no se plantean estrategias claras para } \\
\text { iniciarlo y sólo piensan que deberían hacerlo. }\end{array}$ \\
\hline Preparación & $\begin{array}{l}\text { En etapa se combina la intención y un plan de } \\
\text { acción enfocado al cambio, pero sin comenzar } \\
\text { a hacerlo. En esta etapa los individuos tienen la } \\
\text { intención de tomar medidas y se comprometen a } \\
\text { efectuar un cambio. Se hacen pequeños cambios, } \\
\text { aunque no cumplan los criterios de abstinencia, } \\
\text { por ejemplo. }\end{array}$ & $\begin{array}{l}\text { En esta etapa se identifica a las personas } \\
\text { que acuden con el profesional para atender e } \\
\text { problema de obesidad y establecen planes } \\
\text { para comenzar un cambio en los hábitos de } \\
\text { alimentación y ejercicio (como disminuir el } \\
\text { consumo de endulzantes y/o establecer una plan } \\
\text { de caminata) en donde pueden identificar que } \\
\text { han comenzado por algo, y aquí dependerá de la } \\
\text { motivación del logro obtenida. }\end{array}$ \\
\hline Acción & $\begin{array}{l}\text { Es la etapa en que los individuos modifican } \\
\text { su comportamiento, experiencias o el medio } \\
\text { ambiente para superar sus problemas. Esta fase } \\
\text { implica los cambios de comportamiento de manera } \\
\text { más abierta; requiere una considerable inversión } \\
\text { de tiempo, energía y de mayor reconocimiento } \\
\text { externo. }\end{array}$ & $\begin{array}{l}\text { En esta etapa se ubica a las personas que } \\
\text { llevan a cabo un régimen alimenticio para no } \\
\text { exceder la cantidad de calorías establecidas por } \\
\text { el nutriólogo, además de realizar actividades } \\
\text { físicas para equilibrar el gasto energético de la } \\
\text { ingesta de alimentos, o toman acciones para } \\
\text { regular dichas condiciones, como equilibrar e } \\
\text { tipo de alimentos cuando no tienen tiempo para } \\
\text { hacer ejercicio }\end{array}$ \\
\hline Mantenimiento & $\begin{array}{l}\text { En esta etapa las personas trabajan para evitar la } \\
\text { recaída y consolidan los logros alcanzados durante } \\
\text { la acción; esta etapa debe ser considerada como } \\
\text { una continuación del cambio, e implica sostener } \\
\text { el esfuerzo por continuar los hábitos que han sido } \\
\text { modificados }\end{array}$ & $\begin{array}{l}\text { En esta etapa se identifican las personas que } \\
\text { han logrado un cambio en su problema de } \\
\text { obesidad, como alcanzar un IMC apropiado; sin } \\
\text { embargo, para mantenerse en esa condición es } \\
\text { importante continuar con las acciones y cuidados; } \\
\text { se ha observado que un factor importante en el } \\
\text { mantenimiento es el reconocimiento de otros } \\
\text { como motivador de sostenimiento de la conducta. }\end{array}$ \\
\hline
\end{tabular}


Tabla 2.

Definición e intervenciones representativas de los procesos de cambio

Proceso Definición/InTERVEnCión
EJEMPLOS RESPECTO AL CAMBIO EN LA ACTIVIDAD FÍSICA Y ALIMENTACIÓN PARA DISMINUIR LA OBESIDAD Incremento de la información de sí mismo Confrontar a la persona respecto a su obesidad y

\begin{tabular}{l|l} 
Aumento de la conciencia & y el problema. Esto se puede lograr \\
mediante observaciones, confrontaciones,
\end{tabular}

\begin{tabular}{l|l} 
Aumento de la conciencia & y el problema. Esto se puede lograr \\
mediante observaciones, confrontaciones,
\end{tabular} \begin{tabular}{|l|l|}
\hline & interpretaciones, biblioterapia. \\
\hline & La evaluación de cómo se siente y piensa
\end{tabular}

las consecuencias de ella en su vida y salud, para fomentar la necesidad de hacer cambios en su alimentación y actividad física.

Autorreevaluación de sí mismo respecto a un problema. Clarificación de valores, las imágenes, la experiencia emocional correctiva.

La persona que ha identificado que tiene obesidad necesita valorar sus experiencias, creencias y emociones relacionadas con su condición. Modificar la valoración de que la comida es placentera ayuda a establecer un cambio, porque puede resignificarla a otra conducta más benéfica.

Un adulto obeso puede mejorar su toma de decisiones respecto al consumo de alimentos saludables para mantener su cambio; para ello se utilizan técnicas de toma de decisiones o resoluciones y/o logoterapia, con la finalidad de incrementar su compromiso, así como identificar los recursos que tiene para hacer el cambio.

La elección y el compromiso de actuar o de creencia en la capacidad de cambiar.

La sustitución de opciones para los Un adolescente obeso que se enfrenta a situaciones problemas de comportamiento. La relajación, desensibilización, afirmación y autoafirmaciones positivas.

ansiedad que desencadenan periodos de ingesta descontrolada puede utilizar técnicas de relajación para contrarrestar la ansiedad y evitar una recaída.

Por ejemplo, se puede instruir a la persona para evitar situaciones de riesgo, como acudir a un

Control de estímulos

Evitar o contrarrestar los estímulos que causan problemas de comportamiento.

buffet, ir a lugares donde no se pueda elegir comida saludable, de modo que pueda reestructurar su entorno para no exponerse a situaciones que no pueda controlar.

Para las personas con obesidad se utiliza el

Recompensarse a sí mismo y ser recompensados por otros para hacer cambios. Los contratos de contingencia, abierta y encubierta, refuerzo, autorrecompensa. autorreforzamiento para que cada vez que cumpla con su objetivo de actividad física, refuerce su conducta con un estímulo agradable, como su música preferida durante una hora.

Que la persona busque un grupo de ayuda donde Estar abierto y confiar los problemas a alguien que se preocupa. Alianza terapéutica, apoyo social, grupos de autoayuda.

pueda compartir sus emociones, problemas, situaciones conflictivas; tener una red de apoyo puede ayudar a reforzar sus logros.

Utilizar un juego de roles en el cual la persona

Experimentar y expresar sentimientos acerca

Alivio dramático de los problemas y soluciones personales. Psicodrama, juegos de rol. pueda expresar sus sentimientos acerca de sus situaciones conflictivas para cambiar sus hábitos alimenticios.

Trabajar con la conciencia de tener hábitos sedentarios, de actividad física y una alimentación desordenada y cómo puede influir en los hábitos de sus hijos propiciando sobrepeso y obesidad.

Formación de empatía, documentales.

El aumento de las opciones adaptativas disponibles para problemas de comportamiento en la sociedad. Toma de conciencia de la representación social de los problemas. La defensa de los derechos, el empoderamiento, las intervenciones políticas.

Una persona con obesidad puede trabajar desde el

Liberación social ente y Norcross (1992), p. 1108. ámbito comunitario para generar conciencia social de las consecuencias físicas y psicológicas de no cambiar los hábitos alimenticios y la actividad física. 
lo transteórico, representa una organización jerárquica de cinco niveles en los que se deben enfocar las evaluaciones o intervenciones psicológicas para generar el cambio; los niveles son los siguientes.

1) Síntomas/situación. Se refiere conductas de cambio establecidas en el patrón de uso u otros síntomas, y los factores micro y macroambientales. En este nivel se pueden integrar elementos ambientales que no permiten que se hagan modificaciones en la alimentación, como el tiempo que la persona invierte cada mañana para trasladarse a su trabajo, disminuye el tiempo que tiene para desayunar, el presupuesto que tiene para sus alimentos, el tiempo disponible para hacer actividad física, el tiempo que está sentado frente a la computadora en su trabajo.

2) Cogniciones desadaptativas. Son los cambios a efectuarse en las expectativas, creencias y autoevaluaciones. Por ejemplo, una persona puede tener creencias erróneas acerca de los alimentos, como que la comida saludable no tiene buen sabor, que son más deliciosas las hamburguesas y las papas fritas que unas verduras salteadas.

3) Conflictos interpersonales. Se refiere a cambios en las interacciones diádicas; esto puede verse reflejado en las interacciones que, por ejemplo los pares, tienen con sus hijos en torno a la alimentación; por ejemplo, las discusiones con el hijo por no comer los vegetales que tiene su comida.

4) Conflictos sistémicos/familiares. Se refiere a los cambios dirigidos en la familia de origen, problemas legales, red de apoyo social, empleo, o alguna institución. En este caso se identifican conflictos que pueden afectar de manera directa el presupuesto familiar; por ejemplo, que alguno de los padres en un hogar pierda su empleo y esto disminuye el ingreso familiar, y por tanto se buscan alimentos que pueden no nutrir de modo adecuado, pero satisfacen con rapidez el hambre y son de mejor sabor.

5) Conflictos intrapersonales. Se enfoca en los cambios dirigidos a los aspectos internos de la persona, como la autoestima, autoconcepto y personalidad, entre otros. En este nivel se debe poner especial atención porque las personas con sobrepeso u obesidad pueden presentar un autoconcepto y autoestima baja.

Esta dimensión de los niveles de cambio que plantea el modelo transteórico es relativamente independiente de las otras dos, porque permite definir un perfil de disposición diferencial hacia el cambio, y con ello optimizar la intervención. De acuerdo con Prochaska y Norcross (2007), los niveles de cambio permiten identificar qué tan compleja será la intervención; por ejemplo, si se presenta un nivel de síntomas/situación, el cambio suele ser más rápido porque el problema es más consciente y contemporáneo, a diferencia de un punto más bajo en la jerarquía de los niveles debido a que los determinantes del problema se alejan de la conciencia del individuo, lo que permite predecir que mientras más profundo sea el nivel de cambio, más larga y compleja será la intervención terapéutica y mayor la resistencia del paciente. En este sentido, una persona con problemas de obesidad que tenga conflictos intrapersonales, situacionales y cognitivos implica una intervención más compleja que la persona que sólo tiene conflictos situacionales o de cogniciones.

Para intervenir en múltiples niveles de cambio es necesario considerar las tres estrategias básicas que proponen Prochaska y Norcross (2007, p. 526-527):

1) Cambio sucesivo de niveles. Inicialmente se enfoca en los síntomas del paciente y las situaciones que mantienen estos síntomas; si los procesos se aplican de modo eficiente en el primer nivel y el paciente progresa hacia los siguientes estadios de cambio, la terapia podrá completarse sin necesidad de trasladarse a un nivel más complejo de análisis. Si este enfoque se muestra ineficaz, el foco de la terapia debe trasladarse a otros niveles, y así sucesivamente hasta lograr el cambio deseado.

2) Nivel clave. Si la evidencia disponible indica un nivel clave de causalidad de un problema y el paciente se compromete a trabajarlo, el terapeuta deberá ocuparse casi de manera exclusiva de ese nivel.

3) Impacto máximo. En algunos casos clínicos complejos es evidente la implicación de múltiples niveles como causa y/o efecto de los problemas del paciente. En este caso se pueden diseñar intervenciones dirigidas a tratar múltiples niveles de cambio para conseguir el máximo impacto de una manera sinérgica en lugar de secuencial.

Con estas dimensiones que hemos revisado se plantea el cómo, cuándo y qué se cambia en una intervención, al permitir integrar una amplia gama de estrategias, instrumentos, procedimientos y herramientas para generar cambios; sin embargo, existen dos herramientas propuestas por otros autores que han Ilegado a tener relevancia teórica y empírica dentro de este modelo; la primera es la entrevista motivacional que apoya la toma de decisiones en el proceso de cambio intencional y mantiene los cambios de comportamiento, identificando los pros y los contras de la conducta problema; esta estrategia - propuesta por Miller y Rollnick (2002) — se puede utilizar de- 
pendiendo de la etapa de cambio en la cual se encuentre el individuo; por ejemplo, para que las personas en etapa de precontemplación avancen hacia la siguiente, encontrando la motivación necesaria para iniciar el cambio.

La segunda estrategia son las expectativas de autoeficacia variable propuesta por Bandura en 1977, que conforman un predictor de éxito en el cambio, porque es un proceso cognitivo de evaluaciones o apreciaciones percibidas que las personas hacen de su competencia para hacer adecuadamente una tarea en una situación específica o ante una situación conflictiva, de modo que las evaluaciones que hace una persona acerca de su capacidad para mantener el control de su alimentación será un predictor de cambio; las personas que se perciben poco eficaces para mantener la dieta tendrán menor éxito, más recaídas o tardarán más en llegar a la etapa de mantenimiento.

\section{Conclusiones}

El objetivo del presente análisis fue detallar y ejemplificar los elementos del modelo transteórico en conductas relacionadas con la reducción de peso, como es la adherencia a una dieta sana y el incremento de actividad física. Lo anterior con la finalidad de ser un apoyo para la implementación de intervenciones basadas en el MT para la reducción de factores de riesgo asociados a la obesidad, enfermedades cardiovasculares y diabetes.

Después de la demostración del modelo es posible establecer que, para efectuar una intervención con personas que requieren una modificación en su alimentación y nivel de actividad física, en primer lugar es necesario identificar la etapa de cambio en que se ubican, porque cada estadio precisa una intervención para cada proceso de cambio; asimismo se sitúa el nivel al cual se hará la transformación comportamental, ya sea en síntomas, cogniciones, aspectos interpersonales, intrapersonales o sistémicos, para obtener una mejor adherencia a la alimentación saludable y a la actividad física constante y repetida.

Revisiones como las hechas por Carvalho et al. (2016) indican que las intervenciones basadas en el MT han resultado eficaces para promover un estilo de vida saludable, incrementando la actividad física, el consumo de frutas, verduras, y disminuir la ingesta de grasas, además de mejorar la sustitución por alimentos menor calóricos. El estudio hace constar que muchos de los estudios utilizaron RCT para aportar evidencia de sus efectos en muestras amplias y con seguimientos a largo plazo, lo cual ayuda a identificar que existe una pérdida de peso. Los estudios reportados incluyen muestras diversas, por lo que ha sido aplicado a niños, adolescentes, adultos y personas mayores que pueden estar saludables, en riesgo de desarrollar una enfer- medad cardiovascular u obesidad, así como en individuos con diabetes y tensión arterial elevada.

Ante los hallazgos de investigación reportados al utilizar el MT en este ámbito de acción, es importante destacar que el uso del modelo supone una ventaja ante otras intervenciones psicológicas para la reducción de peso, en tanto que identificar la etapa de cambio en que se encuentra cada individuo permite diseñar acciones específicas para ellos, al contrario de las intervenciones genéricas que se aplican a las personas sin tener en cuenta su motivación para el cambio, como en el caso de Carvalho et al. (2015), que proporcionaron intervenciones específicas para dos grupos de participantes; en primer lugar, aquellos en etapas de contemplación y preparación, y en segundo lugar para quien estaba en acción y mantenimiento; los resultados apuntan hacia una disminución de peso, IMC y la ingesta de alimentos altos en calorías y grasas, lo cual destaca la bondad del uso del MT para intervenciones exitosas con diferentes grupos de personas.

En este mismo sentido la evidencia ha inferido otras variables que pueden caracterizar al individuo en cada etapa de cambio, aludiendo a una mayor motivación en los que están en etapas más activas, como la acción y el mantenimiento, así como menor autoeficacia en aquellos en etapas contemplativas, así como una mayor percepción de barreras en las personas en precontemplación; este tipo de aportes proporcionan un mayor margen de intervención con distintos tipos de personas (Aspano, Lobato, Leyton, Batista y Jiménez, 2016; Jiménez, Moreno, Leyton y Claver, 2015).

Como se indicó en el escrito, un elemento importante y complementario del MT es la autoeficacia que evidencia la confianza que tiene un individuo para controlar los riesgos asociados a la conducta de cambio y progresar en la modificación de la conducta objetivo (Prochaska y Velicer, 1997). Los estudios han mostrado el valor predictor de la autoeficacia y el balance decisional para moverse a una etapa de cambio más activa y mantener los cambios (Ma, Betts, Horacek, Georgiou, White y Nitzke, 2002; Marcus, Selby, Niaura y Rossi, 1992).

Otro aspecto importante que no se puede dejar de lado es la propuesta de integrar la entrevista motivacional, como una estrategia terapéutica que se usa como detonante del cambio, la cual busca iniciar un cambio de conducta ayudando a resolver su ambivalencia con la expresión de empatía, el desarrollo de discrepancias, el movimiento de la resistencia y el apoyo a la autoeficacia, sostenida por creencias basadas en experiencias anteriores o a veces sólo en ideas irracionales; en ambos casos la entrevista motivacional puede contribuir a cuestionar esas creencias y a identificar recursos; estos aspectos han 
sido benéficos en el MT para avanzar hacia una nueva etapa de cambio, y con ello lograr mayor efectividad de progreso en diversos problemas de salud (DiClemente y Velasquez, 2002; Rivera, Villouta y llabaca, 2008; Tas, Sevig y Gungormus, 2016).

Como corolario se puede establecer que el MT es efectivo para el incremento de la actividad física y la mejora de una dieta saludable; sin embargo, es necesario aportar mayor evidencia de la intervenciones basadas en el modelo en países latinoamericanos, en tanto que la mayor parte de la investigación es reportada por países como Estados Unidos, Canadá, y Reino Unido, para establecer si el modelo es viable en poblaciones de nivel socioeconómico más bajo, donde el acceso a los servicios de salud es limitado y existen aspectos culturales que pueden impactar en la intervención.

\section{Referencias}

Aspano, I., Lobato, S., Leyton, M., Batista, M. \& Jiménez, R. (2016). Predicción de la motivación en las etapas de cambio de ejercicio más activo. RETOS. Nuevas Tendencias en Educación Física, Deporte y Recreación, (30), 87-91.

Cabrera, G. A. (2000). El modelo transteórico del comportamiento en salud. Revista Facultad Nacional de Salud Pública, 18(2), 129-138. Recuperado de http://www.redalyc.org/ pdf/120/12018210.pdf

Carvalho, M., Mingoti, S. A., Cardoso, C. S., Deus, R., \& Souza A. C. (2015). Intervention based on Transtheoretical Model Promotes Anthropometric and Nutritional Improvements-A randomized controlled trial. Eating behaviors, 17, 37-44

Carvalho M., Bragunci Bedeschi, L., Dos Santos, L. C. \& Souza A. C. (2016). Interventions directed at eating habits and physical activity using the Transtheoretical Model: a systematic review. Nutricion hospitalaria, 33(5), 1194-1204.

Coombes Y. \& McPherson K. (1996). Review of Health Related Models of Behavior Change: A Report Prepared for the Department of Health in England. London: Health promotion sciences unit of London School of Hygiene end Tropical Medicine.

Crouch, T. B., DiClemente, C. C. \& Pitts, S. C. (2015). End-oftreatment abstinence self-efficacy, behavioral processes of change, and posttreatment drinking outcomes in Project MATCH. Psychology of Addictive Behaviors, 29(3), 706.

Díaz, J. (2001). El modelo de los estadios de cambio en la recuperación de las conductas adictivas. Escritos de psicología, 5, 21-35.

DiClemente, C. \& Prochaska, J. (1982). Self-change and therapy change of smoking behavior: A comparison of processes of change in cessation and maintenance. Addictive behaviors, 7(2), 133-142.

DiClemente, C. C. \& Velasquez, M. M. (2002). Motivational interviewing and the stages of change. Motivational interviewing: Preparing people for change, 2, 201-216.

Gökbayrak, N. S., Paiva, A. L., Blissmer, B. J. \& Prochaska,
J. O. (2015). Predictors of relapse among smokers: transtheoretical effort variables, demographics, and smoking severity. Addictive Behaviors, 42, 176-179.

Greene, G., Redding, C., Prochaska, J., Paiva, A., Rossi, J., Velicer, W. \& Robbins, M. (2013). Baseline transtheoretical and dietary behavioral predictors of dietary fat moderation over 12 and 24months. Eating Behaviors, 14(3), 255-262.

Guo, S. E., Chang, Y. H., Fu, C. H. \& Hsu, M. H. (2016). The Effects of Transtheoretical Model-Based Interventions on Smoking Cessation in Taiwan: A Longitudinal Study. Recuperado de http://www.nursinglibrary.org/vhl/ bitstream/10755/616530/1/64 Guo S p78796 1.pdf

Jiménez, R., Moreno, B., Leyton, M. \& Claver, F. (2015). Motivación y estadios de cambio para el ejercicio físico en adolescentes. Revista Latinoamericana de Psicología, 47(3), 196-204.

Lipschitz, J., Yusufov, M., Paiva, A., Redding, C., Rossi, J., Johnson, S. \& Prochaska, J. (2015). Transtheoretical Principles and Processes for Adopting Physical Activity: A Longitudinal 24-Month Comparison of Maintainers, Relapsers, and Nonchangers. Journal of Sport \& Exercise Psychology, 37(6), 592-606.

Ma, J., Betts, N., Horacek, T., Georgiou, C., White, A. \& Nitzke, S. (2002). The importance of decisional balance and self-efficacy in relation to stages of change for fruit and vegetable intakes by young adults. American Journal of Health Promotion, 16(3), 157-166.

Marcus, B., Selby, V., Niaura, R. \& Rossi, J. (1992). Self-Efficacy and the Stages of Exercise Behavior Change. Research Quarterly for Exercise and Sport, 63(1), 60-66. doi: 10.1080/02701367.1992.10607557

Márquez-lbáñez, B., Armendáriz-Anguiano, A. L., BacardíGascón, M. \& Jiménez-Cruz, A. (2008). Review of controled clinical trials of behavioral treatment for obesity. Nutrición Hospitalaria, 23(1), 1-5. Recuperado de http:// scielo.isciii.es/scielo.php?script=sci_arttext\&pid=S0212$16112008000100001 \& \operatorname{lng}=\mathrm{es} \&$ tlng=en.

Miller, W. \& Rollnick, S. (2002). Motivational interviewing: Preparing people for change Guilford. New York.

Organización Mundial delaSalud[OMS](2017). Noncommunicable Diseases Progress Monitor. Ginebra:Suiza

Prochaska, J. (2013). Transtheoretical model of behavior change Encyclopedia of behavioral medicine (pp. 1997-2000): Springer.

Prochaska, J., DiClemente, C. \& Norcross, J. (1992). In Search of How People Change: Applications to Addictive Behaviors. American Psychologist, 47(9), 1102-1114.

Prochaska, J. \& Marcus, B. (1994). The transtheoretical model: Applications to exercise. In R. Dishman (ed.), Advances in exercise adherence (pp. 161-180). Champaign: Human Kinetics Publishers.

Prochaska, J. \& Norcross, J. (2007). Comparative conclusions: toward a transtheoretical therapy. In J. Prochaska \& J. Norcross (eds.), Systems of psychotherapy: A transtheoretical analysis (pp. 487-528). Australia: Brooks Cole.

Prochaska, J. \& Velicer, W. (1997). The transtheoretical model of health behavior change. American Journal of Health Promotion, 12(1), 38-48. 
Prochaska, J., Norcross, J. \& DiClemente, C. (2013). Applying the stages of change. Psychotherapy in Australia, 19(2), 10.

Prochaska, J., Velicer, W., Rossi, J., Goldstein, M., Marcus, B., Rakowski, W. \& Rossi, S. (1994). Stages of change and decisional balance for 12 problem behaviors. Health Psychology (13), 39-46.

Rivera, S., Villouta, M.F. \& Ilabaca, A. (2008). Entrevista motivacional: ¿Cuál es su efectividad en problemas prevalentes de la atención primaria?. Atención Primaria, 40(5), 257-261. Doi:10.1157/13120020

Rojas-Russell, M. E. (2009). Factores psicosociales asociados a la actividad física en adultos jóvenes: Un estudio transversal. Tipica, Boletín Electrónico de Salud Escolar. 5(2), 136-144. Recuperado de https://www.researchgate. net/profile/Mario_Rojas-Russell/publication/234076496_ Factores Psicosociales Asociados a la Actividad Fisica en Adultos Jovenes Un estudio transversal
Psychosocial Factors Associated to the Physical Activity in Young Adults A cross-sectional study/ links/02bfe50edd154a66f3000000.pdf

Sánchez, A., García, F. \& Landabaso, V. (1998). Participación en actividad física de una muestra universitaria a partir del modelo de las etapas de cambio en el ejercicio físico: Un estudio piloto. Revista de Psicología del Deporte, 7(2), 233-245. Recuperado de: http://ddd.uab.cat/pub/ revpsidep/19885636v7n2/19885636v7n2p233.pdf

Spencer, L., Wharton, C., Moyle, S. \& Adams, T. (2007). The transtheoretical model as applied to dietary behavior and outcomes. Nutrition Research Reviews, 20(01), 46-73.

Tas, F., Sevig, E. U. \& Gungormus, Z. (2016). Use of motivational interview technique with transtheoretical model for behavioral change in smoking addiction. Psikiyatride Guncel Yaklasimlar, 8(4), 380-393. 


\section{Meta-Análisis del Artículo}




\section{Dimensión Cuantitativa}

\section{Perfil de Evaluación entre pares}
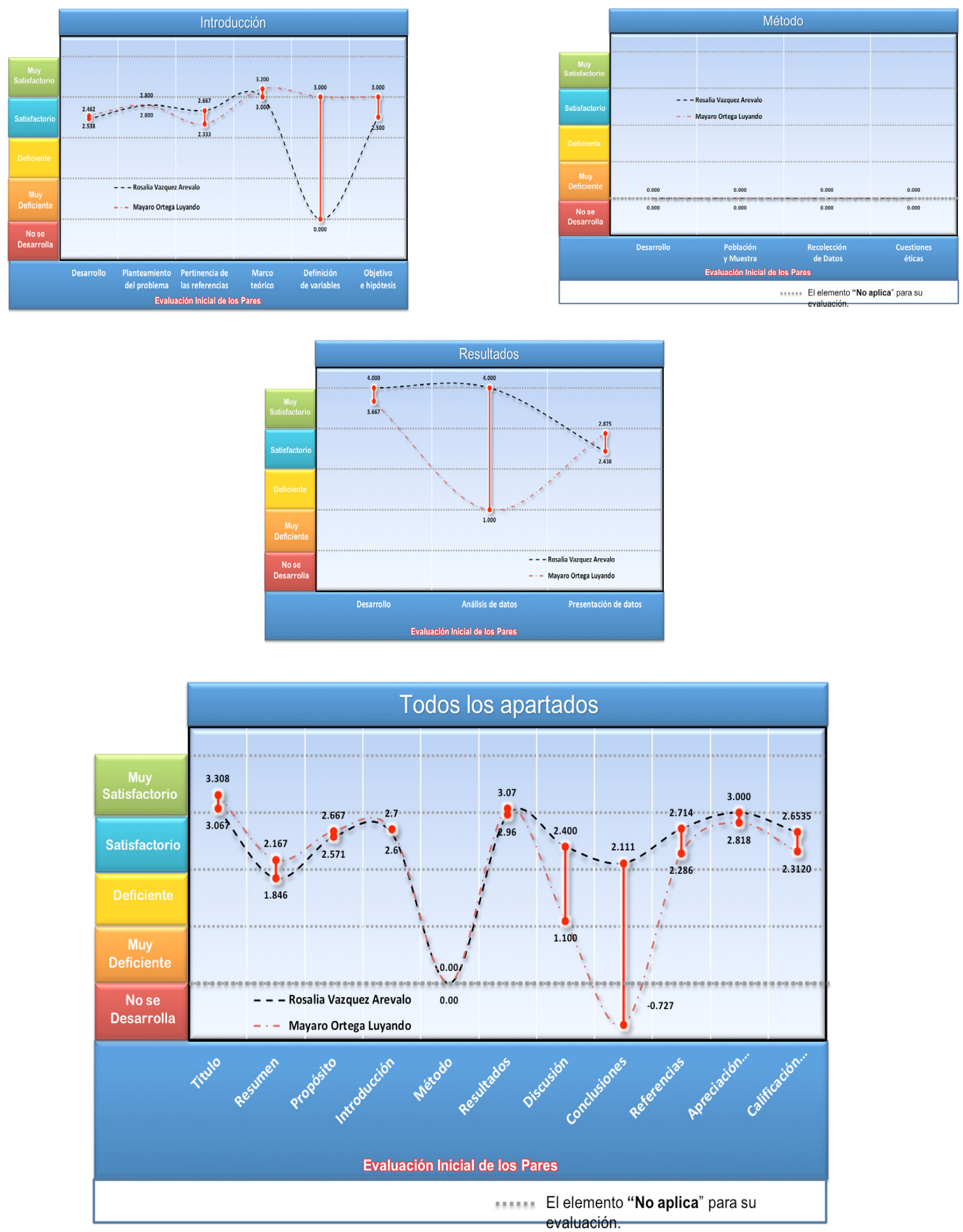


\section{Índice de Concordancia}

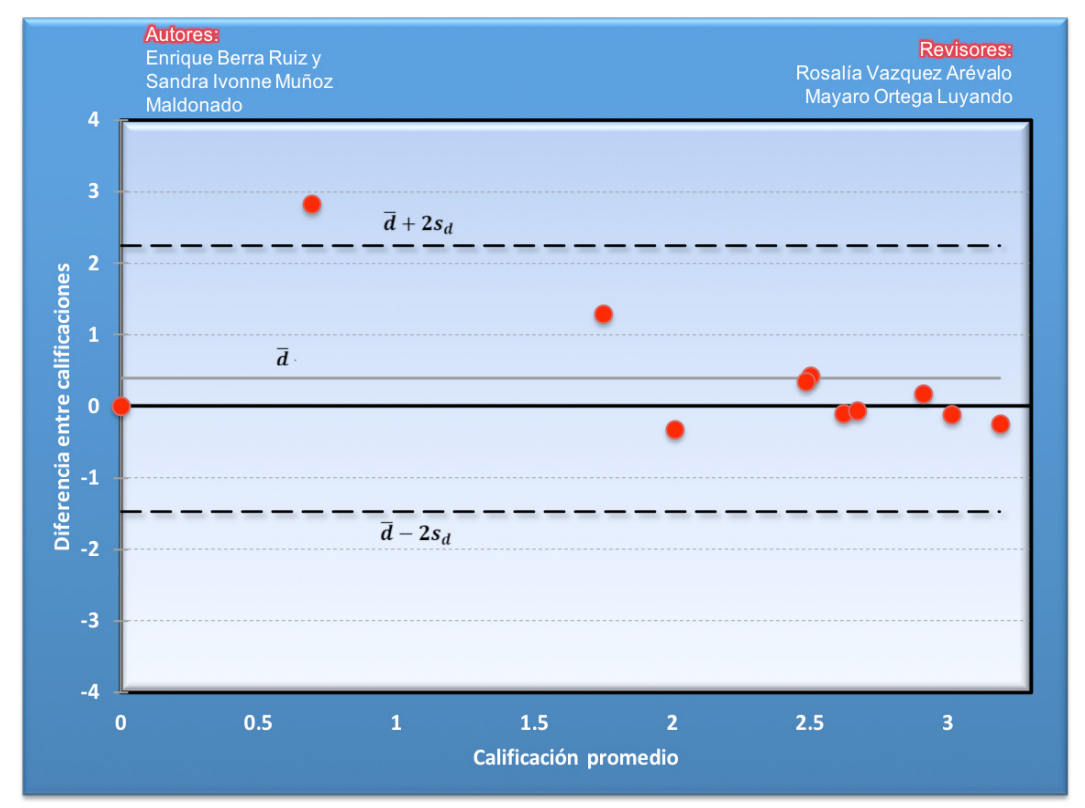

Índice de Acuerdo

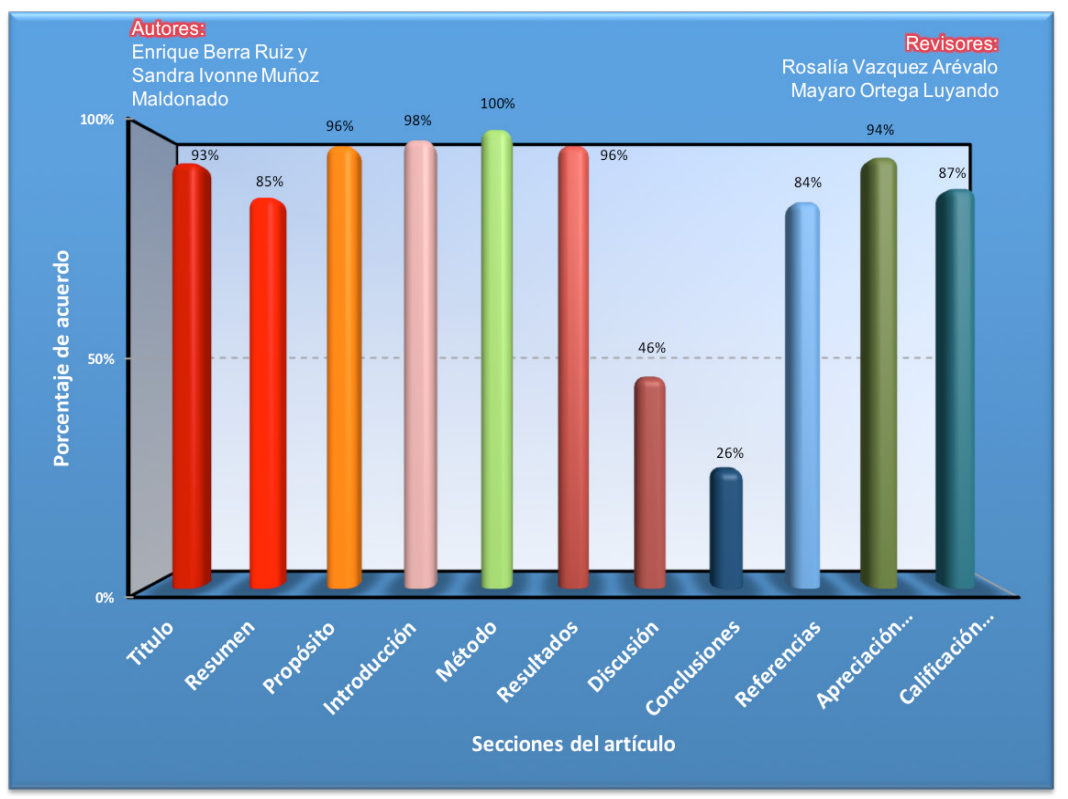




\begin{tabular}{|c|c|}
\hline Revisor 1 & Revisor 2 \\
\hline Rosalia Vazquez Arevalo & Mayaro Ortega Luyando \\
\hline \multicolumn{2}{|c|}{ Título/Autoría } \\
\hline $\begin{array}{l}\text { Propiamente se habla de la reducción de peso, por- } \\
\text { que no se aborda la obesidad, ya que esta puede tener } \\
\text { diferentes génesis. Y estamos ante un caso hipotético } \\
\text { que se supone que la obesidad es consecuencia de un } \\
\text { alto consumo de alimentos. Sugiero cambiar el título } \\
\text { a reducción de peso. EL MODELO TRANSTEORICO } \\
\text { APLICADO en el CAMBIO DE CONDUCTAS RE- } \\
\text { LACIONADAS CON LA REDUCCIÓN DEL PESO } \\
\text { CORPORAL. O algo parecido }\end{array}$ & $\begin{array}{l}\text { Dado que el contenido del artículo refiere a la aplica- } \\
\text { ción del Modelo Transteórico (MT) a dimensiones más } \\
\text { allá de las conductuales (p. ej. sociales), se recomienda } \\
\text { que el título no se centre únicamente en el "cambio de } \\
\text { conductas". Se sugiere el siguiente título: "EL MODE- } \\
\text { LO TRANSTEÓRICO APLICADO AL TRATAMIEN- } \\
\text { TO DE LA OBESIDAD" }\end{array}$ \\
\hline
\end{tabular}

\section{Resumen}

El resumen debe re-elaborarse, no contiene el objetivo, ni qué se hizo en el trabajo, ni las conclusiones

Falta mencionar las principales aportaciones del Modelo Transteórico aplicado a la obesidad y conclusiones generales.

\section{Próposito del Estudio}

Debería modificarse a reducción de peso, en eso no es claro. También debe explicar el ejercicio que se pretende hacer. Poner un objetivo, que sea el mismo en el resumen, introducción y discusión.
No hay una justificación concreta en la que se destaque la relevancia de incluir el MT en el tratamiento de la obesidad.

\section{Introducción}

Dado que es un trabajo teórico se justificar el objetivo, en este caso la utilización del Modelo en las conductas de reducción de peso. Y dado que este es tema, debería aparecer el tema de la obesidad, o la reducción de peso, y porque esta propuesta, ya ha sido explorada antes, etc.
No hay un apartado que establezca claramente inconsistencias en la literatura respecto a la utilización de otros modelos teóricos aplicados al tratamiento de la obesidad. Es necesario que se contextualice que México ocupa los primeros lugares en obesidad infantil y de adultos, asimismo que se mencione qué tipo de intervenciones se llevan a cabo actualmente y cuál ha sido su efecto en la población. En el objetivo se destaca que las variables a explicar son: "la actividad física" y "la alimentación", sin embargo, en el desarrollo del manuscrito se resaltan aspectos de ajuste psicológico y sociales. Se recomienda homologar ambos apartados 


\begin{tabular}{|c|c|}
\hline Revisor 1 & Revisor 2 \\
\hline \multicolumn{2}{|c|}{ Método } \\
\hline $\begin{array}{l}\text { El trabajo refiere una propuesta hipotética de aplica- } \\
\text { ción del modelo Transteórico aplicado a la reducción } \\
\text { de peso corporal. Si bien no tiene los apartado de una } \\
\text { investigación como tal, se esperaría que hubiera un } \\
\text { apartado para explicar qué se va hacer y cómo. Previa } \\
\text { al desarrollo del modelo. }\end{array}$ & $\begin{array}{l}\text { Dado que es un artículo teórico, no hay comentarios en } \\
\text { esta sección. }\end{array}$ \\
\hline \multicolumn{2}{|c|}{ Resultados } \\
\hline $\begin{array}{l}\text { No hay grandes sugerencias, se señalan algunas breves en } \\
\text { el texto. }\end{array}$ & $\begin{array}{l}\text { El objetivo del artículo fue: Ejemplificar el abordaje del } \\
\text { MT en cada uno de los elementos que lo conforman res- } \\
\text { pecto a la actividad física y la alimentación que conduce } \\
\text { a la obesidad y sobrepeso... Sin embargo la Tabla } 1 \text { donde } \\
\text { se ejemplifica el modelo es muy ambigua, esto es, en la } \\
\text { sección de Acción donde se documenta "...podemos ubi- } \\
\text { car a las personas que llevan un régimen alimenticio para } \\
\text { no exceder la cantidad de calorías establecidas...” ¿A qué } \\
\text { se refiere con exceder?, ¿se aplicará el mismo tratamiento } \\
\text { para aquellos que excedan } 10 \text { o } 1000 \text { calorías?, ¿cuál será } \\
\text { el procedimiento a seguir con estas personas?, ¿cómo se } \\
\text { determinará el exceso de calorías ingerido? Asimismo en } \\
\text { la declaración "además de realizar actividades físicas para } \\
\text { equilibrar el gasto energético de la ingesta de los alimen- } \\
\text { tos" ¿Qué tipo de actividad física se realizará?, ¿el psicólo- } \\
\text { go supervisará esta actividad o será un médico?, nueva- } \\
\text { mente ¿cómo se llevará el control de la actividad física, a } \\
\text { través de un acelerómetro o por medio del auto-registro? } \\
\text { En la sección de Preparación, ¿cómo se medirá la moti- } \\
\text { vación del logro obtenido? Se sugiere que la ejemplifica- } \\
\text { ción de los estadios provea información más concreta, y } \\
\text { de ser posible, se especifique qué instrumentos se podrían } \\
\text { utilizar para generar evidencia empírica que sugieran la } \\
\text { efectividad del modelo en las conductas relacionadas a la } \\
\text { obesidad. }\end{array}$ \\
\hline
\end{tabular}




\begin{tabular}{|c|c|}
\hline Revisor 1 & Revisor 2 \\
\hline \multicolumn{2}{|c|}{ Discusión } \\
\hline $\begin{array}{l}\text { Propiamente este apartado no existe, debiera discutirse } \\
\text { aquí los modelos que se han realizado en el área con } \\
\text { el tema u otros temas, pero que adaptaciones propo- } \\
\text { nen. }\end{array}$ & $\begin{array}{l}\text { 1. No hay contrastación con otras teorías ni se desta- } \\
\text { ca la diferencia con el tratamiento cognitivo conductual } \\
\text { (TCC), sobre todo cuando los niveles de cambio que pro- } \\
\text { pone el MT tienen tanta similitud con la TCC. } 2 \text {. Es nece- } \\
\text { sario hacer énfasis con argumentos sólidos por qué este } \\
\text { modelo no debe ser considerado como una visión ecléc- } \\
\text { tica, no es suficiente con mencionar que el modelo posee } \\
\text { "flexibilidad teórica" p.9. Debe retomarse este punto en la } \\
\text { discusión. 3. No hay una contextualización del uso de este } \\
\text { modelo en población mexicana, ¿qué tanta adherencia al } \\
\text { tratamiento aportaría el MT siendo que México ocupa los } \\
\text { primeros lugares a nivel mundial en obesidad?, ¿en qué } \\
\text { escenarios sería ideal la aplicación del MT, en hospitales, } \\
\text { consulta privada, escuelas? } 4 \text {. En el último párrafo cuan- } \\
\text { do se menciona que el MT es efectivo no se cita ningún } \\
\text { estudio que respalde esta afirmación. Se recomienda que } \\
\text { se citen estudios en donde se incluya el tamaño del efecto } \\
\text { para evidenciar la magnitud del cambio a partir del MT. } \\
\text { 5. Finalmente se menciona que el MT es una herramienta } \\
\text { igualmente útil para tratar adicciones que para el uso de } \\
\text { protector solar, ¿cuál es la evidencia detrás de esto? al ser } \\
\text { las adicciones una entidad nosológica y el uso del pro- } \\
\text { tector solar una conducta preventiva, ¿podrían equipa- } \\
\text { rase los tratamientos? }\end{array}$ \\
\hline \multicolumn{2}{|c|}{ Conclusiones } \\
\hline $\begin{array}{l}\text { Faltó análisis, se olvidan de la propuesta inicial, y se } \\
\text { dice que la teoría es muy buena, pero el trabajo tiene un } \\
\text { objetivo, una propuesta. }\end{array}$ & $\begin{array}{l}\text { Se sugiere integrar una sección de limitaciones y de suge- } \\
\text { rencias a futuras investigaciones. No se reflejó la } \\
\text { conclusión en este apartado. }\end{array}$ \\
\hline \multicolumn{2}{|c|}{ Referencias } \\
\hline Actualizar referencias & $\begin{array}{l}\text { Las referencias no están de acuerdo al estilo APA y algu- } \\
\text { nas están dudosamente referenciadas como es el caso de } \\
\text { Prochaska, 2013. En la página 18, segundo párrafo de la } \\
\text { discusión la cita es (Ma et al., 2002) ya que son seis autores. }\end{array}$ \\
\hline
\end{tabular}


